\title{
Next-Generation Optical Access Networks
}

\author{
Leonid G. Kazovsky, Fellow, IEEE, Fellow, OSA, Wei-Tao Shaw, \\ David Gutierrez, Ning Cheng, and Shing-Wa Wong
}

(Invited Paper)

\begin{abstract}
The main bandwidth bottleneck in today's networks is in the access segment. To address that bottleneck, broadband fiber access technologies such as passive optical networks (PONs) are an indispensable solution. The industry has selected timedivision multiplexing (TDM) for current PON deployments. To satisfy future bandwidth demands, however, next-generation PON systems are being investigated to provide even higher performance. In this paper, we first review current TDM-PONs; we designate them as generation $\mathrm{C}$. Next, we review next-generation PON systems, which we categorize into $C+1$ and $C+2$ generations. We expect $\mathrm{C}+1$ systems to provide economic near-term bandwidth upgrade by overlaying new services on current TDM-PONs. For the long term, $\mathrm{C}+2$ systems will provide more dramatic system improvement using wavelength division multiplexing technologies. Some $\mathrm{C}+2$ architectures require new infrastructures and/or equipment, whereas others employ a more evolutionary approach. We also review key enabling components and technologies for $\mathrm{C}+1$ and $\mathrm{C}+2$ generations and point out important topics for future research.
\end{abstract}

Index Terms-Access networks, passive optical network (PON), time division multiplexing (TDM), wavelength division multiplexing (WDM).

\section{Access Technologies: An Overview}

D UE TO advances in photonics technologies and a worldwide deployment of optical fibers, core transport networks have experienced an extraordinary increase in transmission capacity during the last decade. Commercial $1-\mathrm{Tb} / \mathrm{s}$ transmission systems can be deployed using off-the-shelf equipment, and state-of-the-art fiber optical transmission technology has reached $10-\mathrm{Tb} / \mathrm{s}$ per single fiber [1]. In the meantime, at the user end, the drastic improvement of the performance of digital electronics and desktop computers has made possible expanding multimedia services such as video on demand, videoconferencing, high-definition TV (HDTV), e-learning, interactive games, voice over IP, and others. As a result, users will require more than $30 \mathrm{Mb} / \mathrm{s}$ of guaranteed bandwidth per user in the near future [2]. However, current copperwire technologies bridging the users and the core networks are

Manuscript received November 13, 2006; revised July 1, 2007. This work was supported in part by the National Science Foundation under Award 0520291 and Award 0627085 and Altera Inc.

The authors are with Photonics and Networking Research Laboratory, Stanford University, Stanford, CA 94305 USA (e-mail: kazovsky@stanford. edu; wtshaw@stanford.edu; degm@stanford.edu; chengn@stanford.edu; shingw@stanford.edu).

Digital Object Identifier 10.1109/JLT.2007.907748
TABLE I

BANDWIDTH/USER AND MAX REACH OF VARIOUS ACCESS TECHNOLOGIES

\begin{tabular}{lll}
\hline \hline Service & Bandwidth/user & Max Reach \\
\hline ADSL & $2 \mathrm{Mb} / \mathrm{s}$ (typical) & $5.5 \mathrm{~km}$ \\
VDSL & $20 \mathrm{Mb} / \mathrm{s}$ (typical) & $1 \mathrm{~km}$ \\
Coax & $2 \mathrm{Mb} / \mathrm{s}^{*}$ & $0.5 \mathrm{~km}$ \\
Wi-Fi & $54 \mathrm{Mb} / \mathrm{s}(\mathrm{max})$ & $0.1 \mathrm{~km}$ \\
WiMax & $28 \mathrm{Mb} / \mathrm{s}(\mathrm{max})$ & $15 \mathrm{~km}$ \\
BPON & $20 \mathrm{Mb} / \mathrm{s}^{*}$ & $20 \mathrm{~km}$ \\
EPON & $60 \mathrm{Mb} / \mathrm{s}^{*}$ & $20 \mathrm{~km}$ \\
GPON & $40 \mathrm{Mb} / \mathrm{s}^{*}$ & $20 \mathrm{~km}$ \\
\hline \hline
\end{tabular}

* Bandwidth depends on the number of users, and the number listed here is typical values.

reaching their bandwidth limits and become the so-called lastmile bottleneck [3].

There is a strong competition for broadband access services between several technologies: digital subscriber loop, coaxial cable, wireless, and FTTx (FTTx stands for fiber to the $x$, where $\mathrm{x}$ stands for home, curb, neighborhood, office, business, premise, user, etc.). ${ }^{1}$ Table I lists the bandwidth (per user) and the reach of these competing technologies. Currently, the dominant access technologies are digital subscriber loop [International Telecommunication Union-Telecommunication Standardization (ITU-T) G.922], coaxial cable (ITU-T J.112/ 122), and wireless access technologies such as Wi-Fi (IEEE 802.11) and WiMax (IEEE 802.16). The bandwidth and/or the physical reach of copper-wire and wireless access technologies, however, is limited due to the physical media constraints shown in Table I. Therefore, to satisfy the ever-increasing bandwidth demand, service providers will need to deploy optical access networks. We are already witnessing a worldwide deployment of optical access networks and a steady increase in the number of FTTx users.

A number of passive optical networks (PONs) have been standardized to provide broadband access services including ATM PON and broadband PON (APON and BPON, respectively; ITU G983), gigabit PON (GPON; ITU G984), and Ethernet PON (EPON; IEEE 802.3ah). These networks employ time-division multiplexing (TDM) to achieve cost effectiveness and have been widely accepted as the current-generation optical access solutions. In the meantime, next-generation PON architectures are being explored to satisfy the future bandwidth

\footnotetext{
${ }^{1}$ In addition to these competing broadband access technologies, there are also some efforts in developing broadband over power line (BPL; for example, the $\mathrm{X} 10$ standard); however, BPL is immature, and its bit rate is much less than that provided by other technologies.
} 


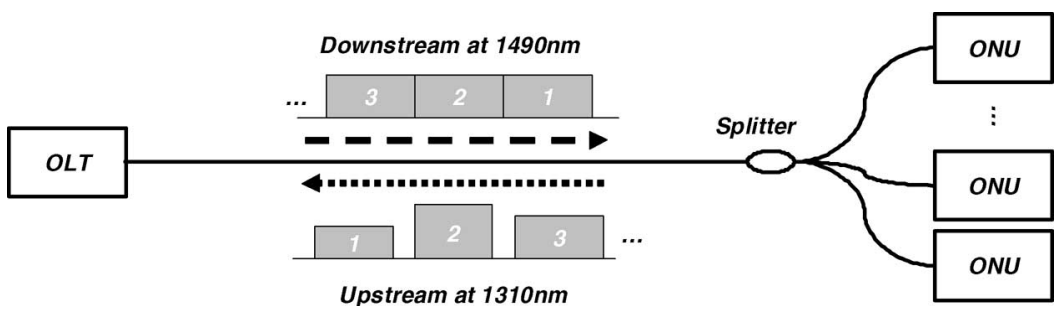

Fig. 1. TDM-PON architecture.

demand. In this paper, we first review the current-generation TDM-PON technologies, then investigate and compare the next-generation PON architectures and enabling technologies, and, finally, point out important topics for future research.

This paper is organized as follows. Section II introduces the optical access networks. Section III reviews the currentgeneration TDM-PONs and their possible enhancements. Section IV introduces and compares the key enabling technologies and architectures for next-generation PONs. Section V discusses the burst-mode transceivers for future PONs. Section VI points out the important issues for future research. Section VII concludes this paper.

\section{IntRoduction to OpticAl ACCESS Networks}

The most straightforward way to provide FTTx, albeit perhaps the most expensive one, is with active point-to-point Ethernet technologies. The basic idea is to replicate the deployments that are common on university and corporate campuses to reach home and small business users, i.e., deploy active equipment that requires electric power, and sometimes air conditioning, throughout the city or suburbs. The main advantage of this approach is that widely available and inexpensive Ethernet equipment can be used and that easier network management is enabled by the active equipment. The downside is the higher cost of installing and maintaining powered equipment cabinets. This approach has had limited success in areas with high population density and was usually deployed when PON technologies were still not mature. Some operators that have deployed this kind of networks are Nippon Telegraph and Telephone Corporation in Japan and FastWeb in Italy.

PON is an important alternative approach. PONs employ a passive fiber tree topology to maximize the coverage, and no active power-consuming elements are deployed in the field. The tree topology allows flexibility and minimizes the number of network splits, thus reducing the optical power loss and increasing the physical reach. Its hardware, bandwidth, and infrastructure are inherently shared among the subscribers; therefore, the cost per user is reduced. Some studies have shown that the operating costs of incumbent local exchange carriers (ILECs) can be actually reduced by aggressively deploying PON since most of the current operating costs of the ILECs come from copper network maintenance [4]. Due to the fast cost drop of optical components, PON has become a feasible broadband access solution and has begun to be deployed and put in service in many countries.

Due to its point-to-multipoint architecture, multiplexing techniques are required in PON to provide multiple-access capability. In TDM-PONs, TDM is used for users to access and share the bandwidth in time domain. The bandwidth provisioned by an optical channel and the hardware in the central office (CO) are, thus, shared among all the users, which is highly desirable to reduce the cost of access networks. Due to its cost effectiveness, the TDM-PON has emerged as the current-generation PON.

The wavelength division multiplexing (WDM) technique can be also employed in PON. For example, each user can be assigned a dedicated wavelength and can enjoy the full bandwidth provisioned. Compared to TDM-PONs, WDM-PONs would provide a much higher bandwidth but would require dedicated hardware for each user, which would result in higher cost. Affordable WDM technology, therefore, is a key issue for WDM-PON systems. Due to their high performance and high cost, WDM techniques are likely to be used in next-generation PON systems but not, as a note, in currently deployed PONs.

Subcarrier multiplexing (SCM) can be also used in PON [5], [6]. Instead of dedicated optical channels, SCM-based PONs use a dedicated electrical subcarrier channel for each user. It enables multiple users to share an optical channel and its associated optical components and, thus, may result in lower cost. The performance (and consequently the cost) of the RF electronics determines the number of users supported and bandwidth for each user.

Optical code division multiplexing (OCDM) has been recently demonstrated as an alternative multiplexing technique for PON [65], [66]. In OCDM-based PONs, users are assigned orthogonal codes, with which each user's data are encoded/decoded into/from an optical pulse sequence. OCDMbased PONs can, thus, provide asynchronous communications and security against unauthorized users. However, the optical encoders/decoders for OCDM are expensive, and the number of users is limited by interference and noise, which will be further discussed in Section III-B.

In this paper, we focus on PON systems based on TDM, WDM, and/or SCM.

\section{Current-Generation PON: TDM-PONs}

A typical TDM-PON system is shown in Fig. 1. Separate lightwaves at 1.5 and $1.3 \mu \mathrm{m}$ are used to carry the traffic from the $\mathrm{CO}$ to an end user (downstream) and from an end user to the CO (upstream), respectively. The optical line terminal (OLT) and the optical network unit (ONU) are deployed as the two ends of the passive optical distribution network (ODN). The ODN consists of feeder and distribution fibers and optical splitter(s). The downstream traffic is continuously broadcast to 
TABLE II

TDM-PON COMPARISON

\begin{tabular}{|c|c|c|c|}
\hline & $\overline{E \text { EPON }}$ & BPON & GPON \\
\hline Standard & $\begin{array}{l}\text { IEEE } 802.3 \\
\text { ah }\end{array}$ & ITU G.983 & ITU G.984 \\
\hline Framing & Ethernet & ATM & GEM/ATM \\
\hline Max Bandwidth & $1 \mathrm{~Gb} / \mathrm{s}$ & $622 \mathrm{Mb} / \mathrm{s}$ & $2.488 \mathrm{~Gb} / \mathrm{s}$ \\
\hline Users / PON & 16 & 32 & 64 \\
\hline $\begin{array}{l}\text { Avg. Bandwidth / } \\
\text { User }\end{array}$ & $60 \mathrm{Mb} / \mathrm{s}$ & $20 \mathrm{Mb} / \mathrm{s}$ & $40 \mathrm{Mb} / \mathrm{s}$ \\
\hline Video & $\mathbf{R F} / \mathbf{I P}$ & $\mathbf{R F}$ & $\mathbf{R F} / \mathbf{I P}$ \\
\hline Estimated Cost & Lowest & Low & Medium \\
\hline
\end{tabular}

all ONUs, and each ONU selects the packets destined to it and discards the packets addressed to other ONUs. In the upstream, each ONU transmits during the time slots that are allocated by the OLT. Due to their burst transmission nature, burst-mode transceivers are required for upstream transmission (the design and challenges of the burst-mode transceivers are discussed in Section V). Since PON may directly connect to end users, the equipment at the ONU is highly cost sensitive. In the ONU, therefore, cheap optical devices such as Fabry-Pérot (FP) lasers are employed.

Table II compares three standardized TDM-PONs: the EPON, the BPON, and the GPON. The maximum bit rate depends on the direction (upstream or downstream) and on the standard; the maximum data rate is $2.488 \mathrm{~Gb} / \mathrm{s}$. Each wavelength is TDM shared among up to 64 users; thus, the average bandwidth per user is limited to a few tens of megabits per second. A third wavelength can be optionally used for downstream video broadcasting.

\section{A. Bandwidth Enhancements of TDM-PONs}

Although TDM-PONs enable significant bandwidth boost as compared to copper-based access networks, their capacity will be exhausted as bandwidth-hungry applications such as HDTV and 3-D video become available. To meet the anticipated demand, two main standardization bodies for optical access networks, i.e., IEEE and ITU-T, are actively developing bit rate (IEEE P802.3av) and wavelength enhancement options, ${ }^{2}$ respectively. These techniques are discussed below.

1) Rate Enhancement of TDM-PONs: To enhance the capacity of TDM-PONs, IEEE has formed a new working group to standardize a $10-\mathrm{Gb} / \mathrm{s}$ version of TDM-PONs. By leveraging the $10-\mathrm{Gb} / \mathrm{s}$ technologies that are widely used in metro and long-haul systems, the goal of a $10-\mathrm{Gb} / \mathrm{s}$ TDM-PON is to achieve an economical bandwidth and service upgrade from current-generation systems. A symmetrical 10-Gb/s TDM-PON system, however, faces two major challenges. First, its upstream bit rate is dependent on the state of upstream burst-mode transmission technology. If the cost of the $10-\mathrm{Gb} / \mathrm{s}$ burst-mode transceiver turns out to be unrealistic for access networks, an asymmetrical system with a lower upstream bit rate might be employed. Second, since the $10-\mathrm{Gb} / \mathrm{s}$ TDM-PON is expected to gradually replace the current TDM-PON service [7], [8], the higher rate and existing signals will coexist until the current

\footnotetext{
${ }^{2} \mathrm{~A}$ wavelength enhancement band is being evaluated in ITU-T G.984.
}

TDM-PON service completely phases out. An economic isolation mechanism, therefore, is required to separate the existing and higher rate signals. In addition, essential technical issues such as extension of current scrambling coding standards and selection of new forward error correction (FEC) for the $10-\mathrm{Gb} / \mathrm{s}$ TDM-PON also need to be addressed.

2) Wavelength Enhancement of TDM-PONs: Since only one wavelength in each direction is used in current TDM-PONs, new services/signals can be launched on different wavelengths to enhance system bandwidth and service. Wavelength enhancement is a promising means for an optical access network to meet the ever-increasing bandwidth need. In BPON, a band between 1539 and $1565 \mathrm{~nm}$ has been reserved for wavelength enhancement (ITU G.983.3 amendment). The overlay of new wavelengths on a working TDM-PON, however, requires network prearrangement or retrofit, which leads to major upgrade costs and presents a serious challenge for the operators. Wavelength enhancement of TDM-PONs is further discussed in Section III-B.

\section{B. Reach Extension and User-Number Increase of TDM-PONs}

In addition to bandwidth enhancements, extending the physical reach and increasing the number of users in a PON are important issues because 1) by supporting more users in a PON system, cost sharing can be further improved, and bandwidth efficiency can be enhanced with higher statistical multiplexing, and 2) extending the physical reach of a PON allows the consolidation of the access and core networks. The consolidation will reduce the number of switching nodes and will result in cost savings for network operation. To increase the physical reach and the splitting ratio of a PON, deploying optical amplifier in the field is preferred over electronic equipment because the former is transparent to traffic formats and bit rates, thus simplifying network management.

SuperPON [9] is an optically amplified PON architecture. In [10], a long reach PON with 1024-way split and 110-km reach running at $10 \mathrm{~Gb} / \mathrm{s}$ has been demonstrated. A standard TDM-PON with extended reach of $135 \mathrm{~km}$ is reported in [11]. Challenges for optically amplified PON include amplified spontaneous emission (ASE) noise, upstream burst-mode transmission, and operating cost associated with active node management.

Besides optical amplifiers, the OCDM technique can be used to extend the physical reach of a PON. Reference [12] demonstrates a PON system with $100-\mathrm{km}$ reach without optical amplifiers in the field. The proposed electrical matched filter design has potential to reduce the cost of the coder/decoder. One challenge for the OCDM PON is the interference and noise as the number of users increases. Efficient OCDM access codes are required to reduce the interference and noise without introducing too much overhead, and the FEC technique can be utilized to combat the interference and noise.

\section{NeXT-Generation PON ARchitectures}

Quite a few next-generation PON systems have been proposed by both academia and industry. Some of them [13]-[16] 


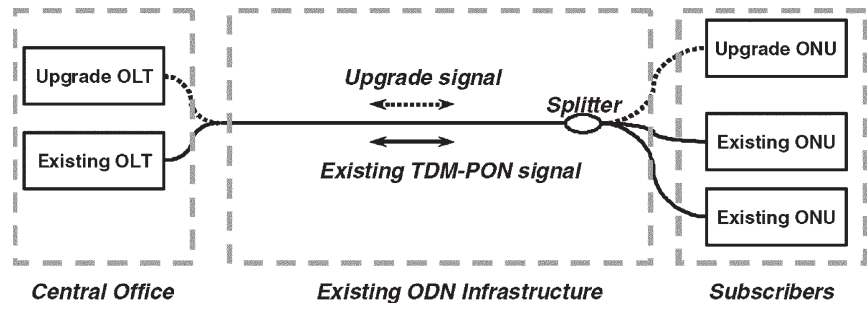

Fig. 2. Generalized architecture of $\mathrm{C}+1$ generation PON system.

focus on providing a graceful bandwidth upgrade from the current-generation TDM-PON system. Near-term bandwidth upgrade can be anticipated due to the following reasons:

1) insufficient bandwidth of the current-generation TDMPON system [7];

2) need to meet higher bandwidth and service demand from certain users, e.g., business users [13];

3) need to enable joint services provided by multiple service providers on a shared access network infrastructure [14].

In essence, the goal of a graceful upgrade process is to allow the bandwidth and services to be upgraded, with the existing service and equipment as intact as possible. Therefore, a smooth upgrade is highly desirable for system operators since the resulting investment is lower and more flexible. To emphasize that these systems enable a smooth upgrade from currentgeneration TDM-PONs in the near future, we categorize them as the $\mathrm{C}+1$ generation $\mathrm{PON}$ system. Most other next-generation PON proposals focus on achieving much higher capacities to satisfy the long-term demand [34]-[51]. We categorize such systems as the $\mathrm{C}+2$ generation PONs. In $\mathrm{C}+2$ systems, more radical new architectures and technologies are usually required to support high capacity, realize cost effectiveness, or facilitate network scalability. Some of the $\mathrm{C}+2$ architectures also facilitate graceful evolutionary approaches [47], [49].

\section{A. $C+1$ Generation PONs}

A generalized $\mathrm{C}+1$ generation PON is illustrated in Fig. 2. Rather than replacing the existing service, the upgrade service is overlaid on a working TDM-PON. As a result, the new and existing signals need to be separated as they are received. To achieve the required signal separation, three techniques have been proposed for $\mathrm{C}+1$ generation systems:

1) launching the upgrade signal at new wavelengths with prearranged or retrofitted network equipment;

2) moving the upgrade signal out of the baseband of the existing signal with the SCM technique;

3) shaping the spectrum of the upgrade signal with the spectral line-coding technique to minimize its interference on the existing signal [14].

The foregoing techniques can be jointly used for cost and performance optimization. We will now describe these enabling techniques and review selected $\mathrm{C}+1 \mathrm{PON}$ architectures.

1) Service Overlay With WDM Techniques: It is fairly straightforward to employ WDM techniques to separate the upgrade and existing signals. References [13] and [15] give examples of launching the upgrade signal on the enhancement band. In [13] and [15], a pair of dense wavelength-division multiplex- ing (DWDM) channels is dedicated to each upgrade user for bidirectional transmissions, as in Fig. 3. A point-to-point link is, thus, formed between the $\mathrm{CO}$ and each user, and each user can enjoy the full bandwidth provisioned by the wavelength pair. Without replacing the passive optical splitter deployed in the field, DWDM filters and multiplexer (MUX)/demultiplexer (DMUX) are employed in the upgrade ONUs and CO, respectively [13], [15]. Furthermore, to separate the upgrade and existing signals in different wavelength bands, coarse wavelength-division multiplexing (CWDM) MUX/DMUX are required in the $\mathrm{CO}$ and the ONUs. In [15], simulations are reported to evaluate the feasibility of the proposed system and address its performance limitations. The most significant noise that is injected into the upgrade DWDM OLT and ONU is due to the adjacent channel crosstalk, which depends on the total number of DWDM channels used. This noise can be reduced by alternating the downstream and upstream wavelength channels in the enhancement band. The performance degradation of the existing service, due to the crosstalk of the upgrade DWDM signals, is not investigated in [15]. Given the characteristics of the commercial CWDM MUX/DMUX and the specified guard band between the existing and upgrade DWDM signals, the resulting performance degradation can be made insignificant.

The main disadvantage of using the WDM technique for system upgrade is that it requires either prearranging or retrofitting the hardware of existing ONUs. The prearrangement or rearrangement requires the ONU module of current-generation TDM-PONs to be equipped with a CWDM MUX/DMUX. The prearrangement, however, is not required in the standards. Therefore, in most cases, network retrofitting will be required a CWDM MUX/DMUX will need to be added in every existing ONU. As TDM-PONs have become prevalent and will need to be upgraded, the retrofitting cost can be considerable because the number of existing ONUs will be large, and their locations will be widely dispersed.

Compared to retrofitting the existing ONUs, the cost for adding a CWDM MUX/DMUX in the CO is acceptable because the number of COs is made smaller, and they are more accessible to system operators. Hence, WDM techniques can be more viable for service overlay in the upstream transmission.

2) Service Overlay With SCM: The upgrade and existing signals can be separated in the electrical domain. In a TDM-PON, nonreturn-to-zero encoding is used for downstream and upstream traffic. The spectral content of the existing signal, therefore, is mostly located in the baseband. To separate from the existing signal, the SCM technique can be used to shift the upgrade signals to RF subcarrier channels.

Reference [16] proposes a smooth upgrade using the combined WDM and SCM techniques for the downstream and upstream transmissions, respectively, as shown in Fig. 4. In [16], the downstream upgrade signal is transmitted at a different wavelength from the existing signal (it is assumed that the required WDM filter is already installed in the existing ONU). In the upstream, both the upgrade and existing signals are transferred on the same wavelength, whereas the signal from each upgrade ONU is continuously transmitted on a dedicated RF subcarrier channel. The capability of simultaneously detecting the baseband and subcarrier signals in the $\mathrm{CO}$ hinges on the 


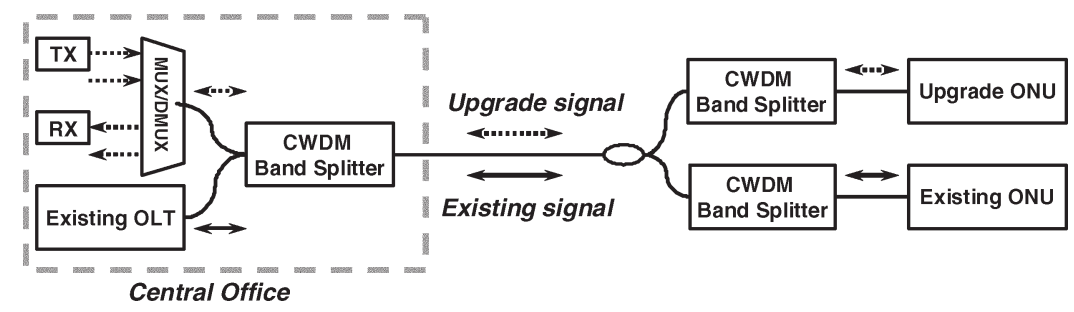

Fig. 3. Service overlay with DWDM technique in downstream and upstream directions.

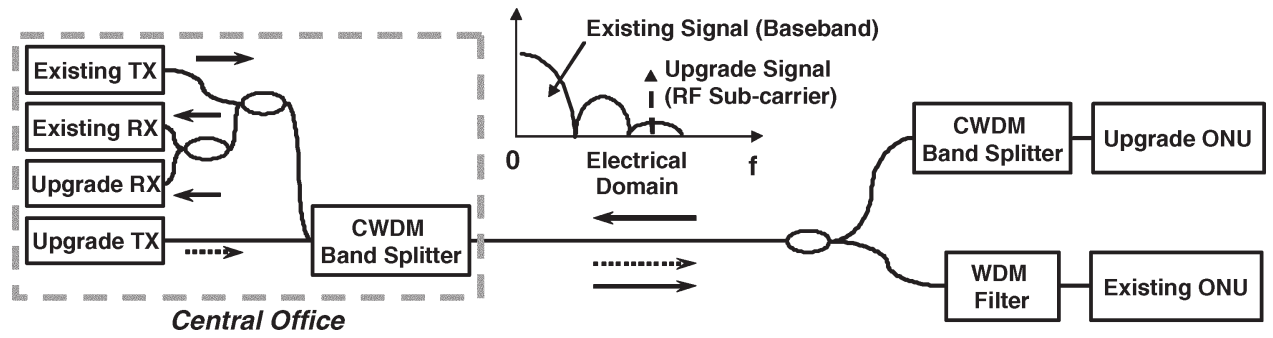

Fig. 4. Service overlay with SCM technique in the upstream direction and WDM technique in the downstream direction.

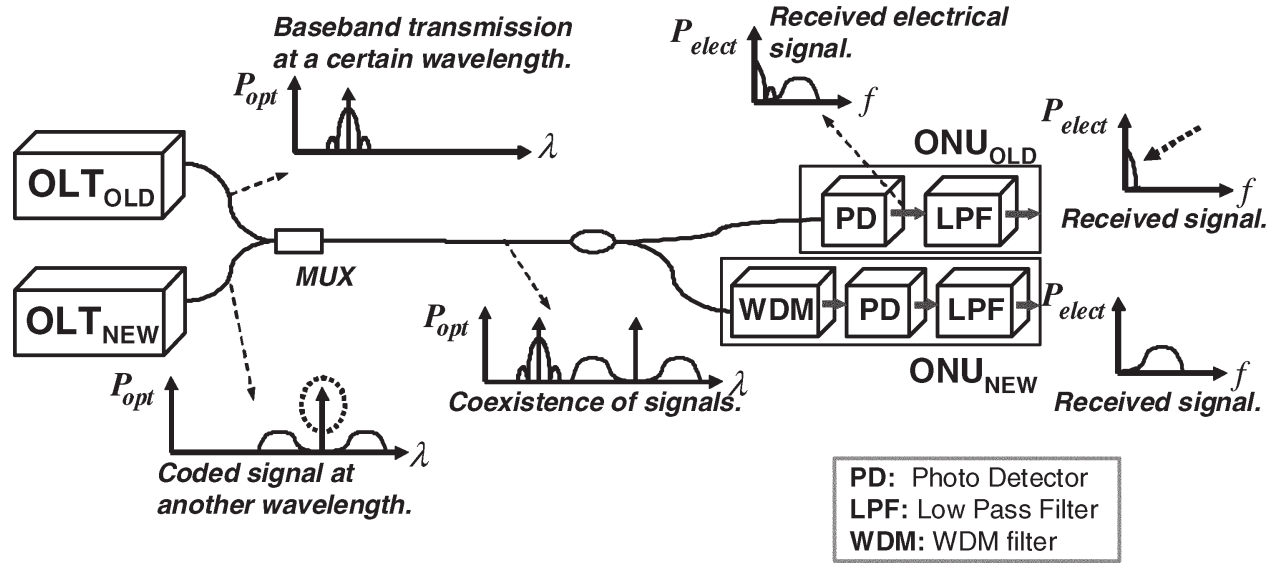

Fig. 5. Service overlay with spectral-shaping line coding technique in the downstream direction and WDM technique in the upstream direction.

ability to separate these signals in the electrical domain. In particular, since the spectrum of the baseband signal slowly falls off with frequency, harmonic lobes will overlap the RF subcarrier channels and interfere with upgrade signals. The interference is dependent on the bit rate of the baseband signal and the RF frequencies of the subcarrier channels. The interference can be suppressed if an electrical low-pass filter is installed in the existing ONU transmitter. The impact of a low-pass filter is investigated in [16]. Installing an electrical low-pass filter, however, will require prearrangement or retrofit of the existing ONUs and will result in increase in upgrade cost.

In addition to RF interference, the optical beat interference (OBI) is another challenge for SCM techniques. OBI occurs when light from two lasers is simultaneously detected by a photodiode. If the lasers have optical frequencies differing by the frequency of one of the subcarrier channels, then the optical beat noise will degrade the signal-to-noise ratio of that channel [17]. To prevent this impairment, various techniques such as broadening the laser linewidth [17], [18], employing optical phase modulation [19] and amplified light-emitting diode [20], and using electrical spread spectrum [21] have been reported.
3) Service Overlay With Spectral-Shaping Line-Coding Technique: Line coding is a technique that tailors the transmission signal to improve or facilitate signal reception [22]-[24]. For example, 8B10B line coding has been widely used to provide high transition density and dc balance, which facilitates the receiver design and clock recovery [25]. Similar to SCM, a spectral-shaping line-coding technique can push the frequency content of the upgrade signal out of the baseband that is occupied by the existing signal. A flexible spectral-shaping linecoding technique that is designed for graceful and cost-effective system grade is reported in [14]. In [14], the suppression of the low-frequency component is achieved by periodically inserting redundant bits into the data bitstream. Unlike the subcarrier technique, the spectral line-coding approach can be implemented in software or firmware and does not impose extra hardware requirements. The proposed algorithm allows flexibility for optimal performance given different system requirements [14]. However, inserting redundant bits will inevitably reduce the transmission efficiency.

The upgrade scenario proposed in [14] is illustrated in Fig. 5. The WDM technique is used for upstream upgrade, the 
TABLE III

COMPARISON OF THE ENABLING TECHNOLOGIES IN C + 1 GENERATION PON

\begin{tabular}{lllll}
\hline \hline & $\begin{array}{c}\text { Hardware } \\
\text { Pre-arrangement } \\
\text { or Retrofit }\end{array}$ & $\begin{array}{c}\text { Transmission } \\
\text { Efficiency }\end{array}$ & Flexibility & Estimated Cost \\
WDM & Required & $100 \%$ & Low & High (Downstream) \\
& Optional & $100 \%$ & Low (Upstream) \\
SCM & Opt & Hedium \\
SSLC & Not required & $<100 \%$ & Low & \\
\hline \hline
\end{tabular}

${ }^{1}$ SSLC: Spectral-Shaping Line Coding

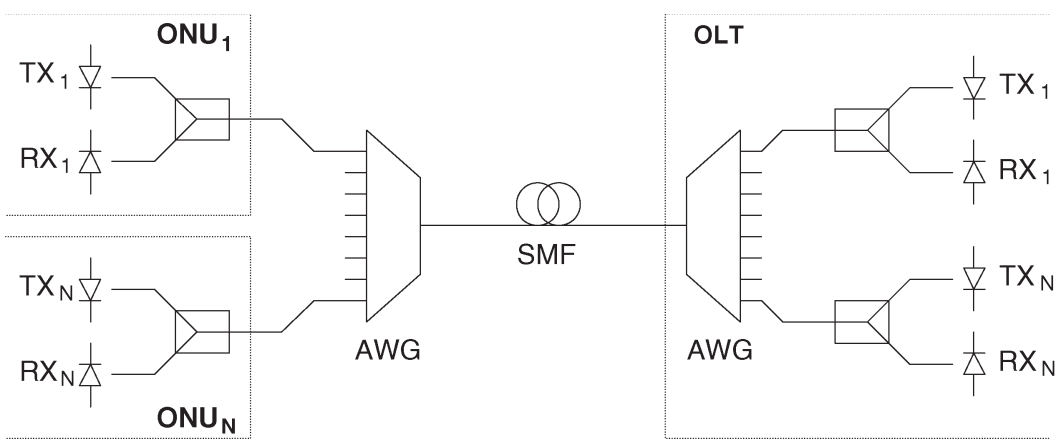

Fig. 6. WDM-PON example.

upgrade signal is transmitted on a different wavelength, and the existing ONUs are left intact. The existing and upgrade signals are separated in the CO by adding a CWDM DMUX, which does not cause a significant cost increase. For the downstream upgrade, instead of retrofitting the existing ONUs by adding narrow-band filters, the proposed spectral-shaping line coding is used to tailor the spectrum of the upgrade high bit-rate signal. As a result, the low-frequency component of the upgrade signal is suppressed. When an existing ONU receives the downstream upgrade signal, an electrical low-pass filter, which is used to filter out the out-of-band noise, would also filter out the upgrade signal. In this way, the interference from the upgrade signal is minimized, and the existing ONUs remain intact. Since the upgrade signal is carried on a different wavelength from the existing signal, WDM filters in upgrade ONUs filter out the existing signal. Note that using electrical bandpass filter in the upgrade ONUs can further improve the rejection of existing baseband signals.

Experimental results reported in [14] compare schemes with different coding efficiency and different spectral overlap with the existing signal. The existing signal is assumed to be $1 \mathrm{GBd} / \mathrm{s}$, and the upgrade signal is $10 \mathrm{GBd} / \mathrm{s}$. The interference of the upgrade signal depends on the coding efficiency; higher efficiency will cause higher interference on the existing signal. For a specific tolerable interference level, the optimal line code can be found, which results in maximum data throughput.

The three technologies enabling service overlay in $\mathrm{C}+1$ generation PONs are summarized in Table III.

\section{B. $C+2$ Generation PONs: Higher Capacity Architectures}

In this section, we describe the $\mathrm{C}+2$ generation of $\mathrm{PON}$ architectures in which the throughput capacity of the network is many times higher than that of current TDM-PONs, e.g., several gigabits per second per user.
Fig. 6 illustrates an example of a basic WDM-PON. On the right, the OLT, which resides at the CO, has an array of transmitters and receivers. Each transmitter-receiver pair is set at the wavelength band of the port of the multiplexing device, in this case, an arrayed waveguide grating (AWG), to which the pair is connected. A feeder fiber connects to this OLT AWG to another AWG in the distribution network. Each port of the second AWG is connected to a different ONU. Each ONU has a passive splitter that is connected, on one end, to a transmitter and, on the other end, to a receiver. In this particular architecture, the receiver needs not be wavelength selective; however, the transmitter needs to be set at a particular wavelength of the AWG port that it is connected to. The distribution network can include several AWGs that are cascaded to increase flexibility, as in [26].

The architecture such as the one described in Fig. 6 is quite expensive for access networks, and having a separate ONU per each PON customer creates an inventory problem. Cost-effective implementation of WDM light sources at the ONU side is necessary for WDM-PONs. Several approaches have been demonstrated for the implementation of colorless ONUs in WDM-PONs, such as spectral sliced broadband light source (BLS), injection-locked FP lasers, and reflective semiconductor optical amplifiers (SOAs). The wavelengths of these light sources are not determined by the gain media, but by external factors - optical filters or injected signals. As a result, the wavelengths are easier to manage without worrying about temperature or aging effects in the ONUs.

For optical transmitters, conventional laser diodes such as FP lasers, DFB/distributed Bragg reflector (DBR) lasers, and vertical cavity surface-emitting lasers (VCSELs) are normally used under direct modulation. Optical receivers generally use p-i-n or avalanche photodiodes for photodetection. Passive splitters can be implemented with fused fibers or planar lightwave circuits, and athermal AWGs are commonly used as 
MUX/DMUX. Operational details of these components are beyond the scope of this paper; the interested reader is referred to [27]. In this section, we discuss some of the most important enabling technologies for $\mathrm{C}+2$ generation PONs and the resulting network architectures.

1) WDM-PONs Based on Tunable Components:

Tunable lasers (TLs): Tunable light sources provide flexibility and reconfigurability for network provisioning, minimize production cost, and reduce the backup stock required. Commonly used options for TLs are external cavity lasers, multisection DFB/DBR lasers, and tunable VCSELs. Due to the cost concerns, it is desirable that TLs can be directly modulated.

An external cavity laser is usually tuned by changing the characteristics of the external cavity, which consists of grating or FP etalon. The tuning ranges (TRs) of external cavity lasers are extremely wide, covering a few hundred nanometers. However, the long cavity length prevents high-speed modulation; therefore, external cavity lasers are not suitable for fiber optic communications. Tuning speed and stability are also issues with external cavity lasers.

Traditional DFB lasers can support high-speed direct modulation and be thermally tuned over a few nanometers. However, tuning speed is limited to the millisecond range. Multisection DFB/DBR lasers usually consist of three or more sections: an active (gain) section, a phase-control section, and a Bragg section. Wavelength tuning is achieved by adjusting the currents in phase-control and Bragg sections. Using multisection DFB/DBR lasers, the tuning speed can reach nanoseconds by current injection, and TRs over tens of nanometers can be achieved. Some multisection DFB/DBR lasers with sampled gratings can be tuned over $100 \mathrm{~nm}$ [28]. The disadvantages of multisection DFB/DBR lasers are mode hopping and complicated electronic control.

VCSELs emerged as a new type of semiconductor lasers in the 1990s. A typical VCSEL consists of a layer of gain medium that is sandwiched between two oppositely doped distributed Bragg reflectors. VCSELs have a potential for lowcost mass production because of simple one-step epitaxy and on-chip testing. However, the development of long-wavelength VCSELs has been hindered by unsatisfactory optical and thermal properties of InP-based III-V materials. New design using different materials and dielectric mirrors has led to successful development of 1.3- and 1.5- $\mu \mathrm{m}$ VCSELs. A primary tuning mechanism for VCSELs uses a microelectromechanical system structure that changes the cavity length through electrostatic control. The tuning speed can be a few microseconds, and the TR can reach 10-20 $\mathrm{nm}$ [29]. As its fabrication matures, VCSELs will be a strong candidate for access networks.

Tunable receivers: A tunable receiver can be implemented using a tunable optical filter and a broadband photodiode. Although this approach provides a simple solution, it can be bulky and costly. As an alternative, complementary metal-oxide-semiconductor-controlled tunable photodetectors with integrated filtering mechanism in the photon-detection process have been reported [30]. The device consists of a few metal-semiconductor-metal detectors laid side by side with interleaved metal fingers. As multiple wavelengths impinge on the photodetector, different wavelengths form different fringe patterns. When a specific biasing pattern is applied to the biasing fingers, the photodetector can select one wavelength while canceling out the rest. Since the wavelength of this device is electronically set, the tuning time is essentially limited by the electronics switching time, which is on the order of nanoseconds. The channel spacing is limited by the coherence length of the laser system and can be much less than the standard 50-GHz DWDM requirement. The detecting algorithm of the device can be applied to either 1550- or 850-nm optical systems. In addition, this device also allows spectral shaping to adapt to specific system applications.

The disadvantage of this device is the scalability of the integrated interferometer. To have satisfactory spectral responses, the device needs to have an integrated interferometer that simultaneously generates a few harmonic interference patterns. This interferometer will become more complicated as the wavelength number in the access network scales up. It, thus, prevents a single device from being applied in a large access network. This limitation can be relaxed by the proper design of access network architectures.

Network architectures: In Fig. 6, we could replace $\mathrm{TX}_{1}$ through $\mathrm{TX}_{N}$ with TLs. This would allow all ONUs to be identical, thus solving the inventory problem that having a different ONU per each customer would create. The TLs would need to cover a wide range of DWDM channels, as many as the range of the AWG MUX/DMUX. The wavelength output of the TL needs to be precise to stay within the AWG port band that it connects to. Thus, TL diodes require expensive temperaturestabilization control circuitry. Although having identical ONUs solves the inventory problem, the cost of these tunable devices at the moment is too high to be cost effective for access. On the other hand, employing TLs in the CO can be economically feasible when they are time shared among users. Such architectures [47], [49] will be discussed later in this paper.

2) WDM-PONs Based on Spectral Slicing:

Spectral sliced BLSs: A comb of optical signals, each with a unique wavelength, can be achieved by slicing the spectrum of a BLS. The BLS can be a superluminescent light-emitting diode [31], erbium-doped fiber amplifiers [32], or an FP laser [33]. For wavelength selection, an AWG, usually deployed at the remote node $(\mathrm{RN})$, will slice a narrow spectrum of the broadband optical signals, and different wavelengths will be selected for different ONUs. If a tunable filter is used, the wavelengths of the optical comb can be tuned.

Network architectures: The spectral slicing technique was first used in [34] for downstream transmission and in [31], [35], and [36] for upstream transmission. Fig. 7 illustrates the BLS using spectral slicing. Each ONU has a BLS that is modulated with upstream data. Upon reaching the WDM MUX/DMUX, the spectrum of each ONU is "sliced." After that, multiple data streams can be multiplexed onto the distribution network fiber. At the OLT, the signals are demultiplexed, and a separate receiver for each ONU is used.

The BLS spectrum should be sufficiently wide compared to the WDM MUX/DMUX, and its output power should be large enough so that after being spectrally sliced (which leads to significant optical power loss, e.g., $10 \mathrm{~dB}$ in [35]), the remaining power will still be enough to traverse the feeder fiber 


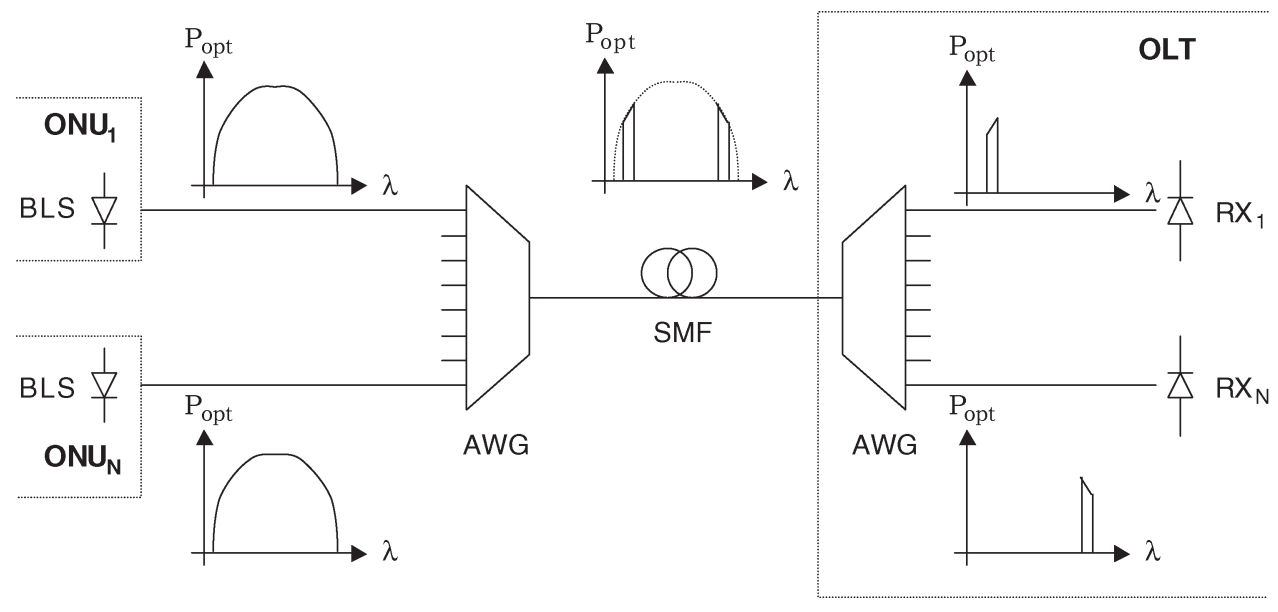

Fig. 7. Upstream transmission using BLS with spectral slicing.

(about $20 \mathrm{~km}$ in current standards). This, added to the coupling, connectors, and device losses, may induce additional loss of up to $20-30 \mathrm{~dB}$ after spectral slicing.

A sharp filtering profile of the WDM MUX/DMUX is necessary to reduce the crosstalk between adjacent channels. Another issue with the AWG is its thermal sensitivity. Since these devices are placed in the outside plant and not powered, their wavelength properties should be insensitive to temperature changes. Theoretical analysis and experimental results [37] have shown that the adjacent-channel crosstalk can be a problem for this technique.

The main advantages of spectral slicing are simple implementation and low cost. The disadvantages are limited modulation speed, low power, and incoherent output that limits the transmission span.

The BLS with spectral slicing brings some limitations to the network topology as well. In particular, the signals from different ONUs share the same spectrum. Therefore, they cannot be combined until after they have been spectrally sliced at the AWG; otherwise, upstream traffic collisions may occur. This reduces the flexibility of the network since some TDM-PON deployments have more than just one splitting point.

3) WDM-PONs Based on Injection Locking:

Injection-locked FP lasers: When an external narrowband optical signal is injected into a multiple-longitudinalmode laser such as an FP laser, the lasing mode can be locked to a single mode. The external optical signal acts as a seed for laser oscillation in the FP cavity; moreover, the mode that is nearest to the peak wavelength of the injected optical signal will be locked to the injected light, and other modes will be suppressed, as shown in Fig. 8 [38]. The injected optical signal can be a spectral sliced BLS (e.g., ASE) [39] or a modulated downstream signal [40]. The advantages of using injectionlocked FP lasers are simplicity and low cost, and the modulation speed can be relatively high. The disadvantages are the limited wavelength locking range, high required power, and questionable stability. Modulation index, laser bias current, and the power of external optical excitation must be carefully chosen to maximize the efficiency of injection-locked FP lasers [41].

Network architectures: Several network architectures have been reported based on injection-locked lasers [33], [42].

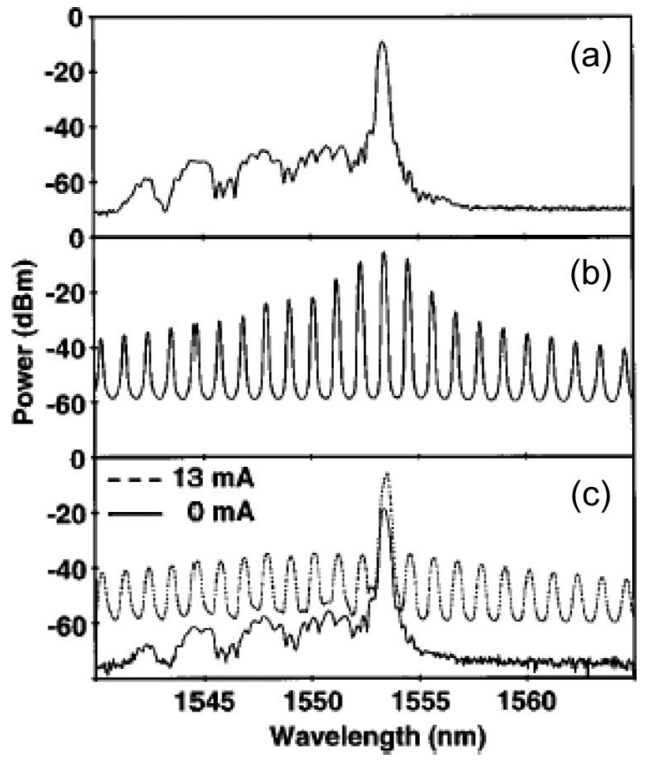

Fig. 8. Injection locking [67]. (a) Injected narrow-band ASE. (b) Fabry-Pérot laser diode (FPLD) output without injection. (c) FPLD output with injection at two bias currents.

Fig. 9 shows an example from [42] both in the downstream and upstream directions. At the CO, a BLS generates optical power that is sent downstream. This optical power is sliced by the remote AWG and is used to injection-lock the ONU FP laser. The locked FP laser is then modulated, and the data are sent in the upstream direction. A circulator at the OLT relays the aggregated upstream signal to a WDM DMUX followed by a set of designated receivers.

In this architecture, higher optical injection power is required for higher data rate to achieve colorless operation [42]. Since the signal is transmitted on the same wavelength as the injection seed light, the BLS power cannot arbitrarily increase because of Rayleigh backscattering and reflections. The scalability of this architecture needs further investigation.

4) WDM-PONs Based on Centralized Light Sources (CLSs):

CLSs: Another alternative for colorless ONUs is to use CLSs at the OLT, as shown in Fig. 10. In this approach, no light source is deployed at the ONUs; the OLT provides optical 


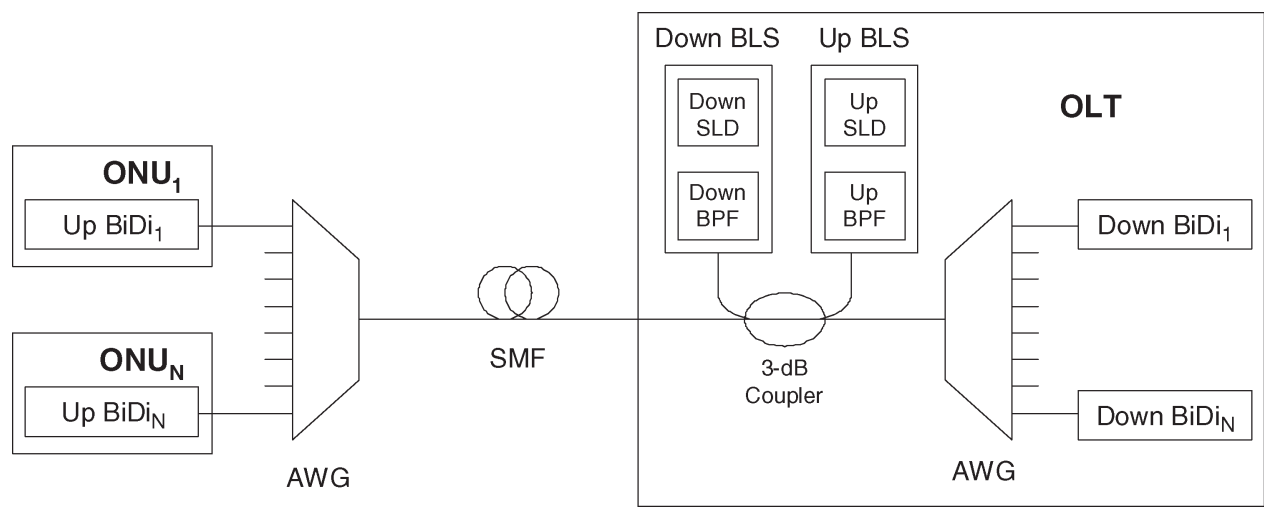

Fig. 9. Bidirectional transmission using injection-locked FPLDs, based on [42].

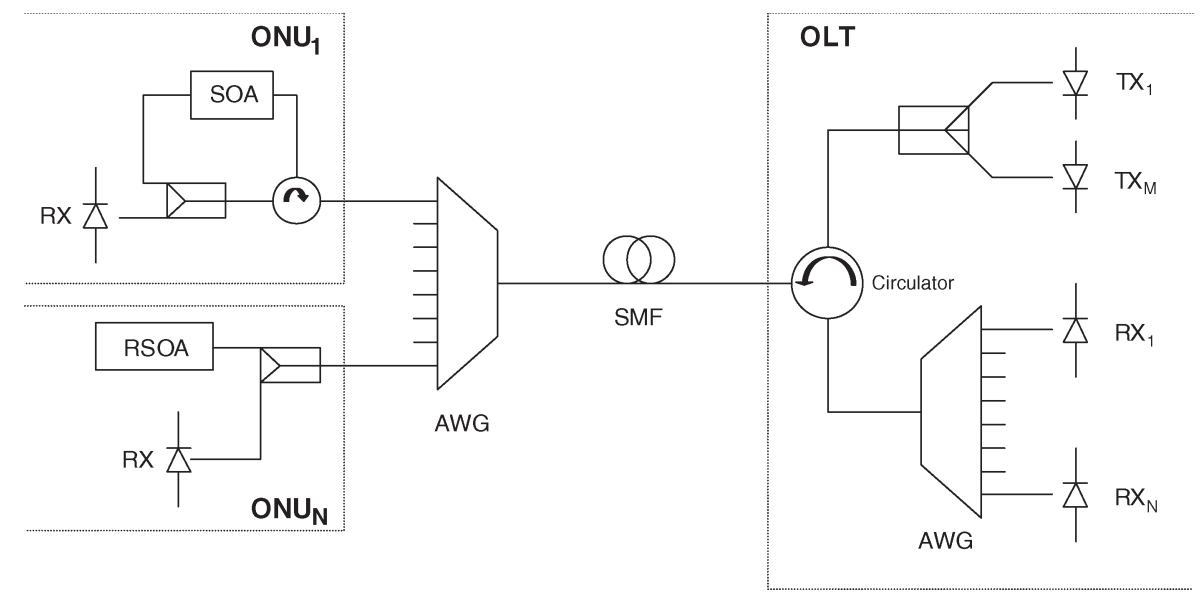

Fig. 10. Upstream transmission using CLS.

signals to ONUs, where the optical signals are modulated with the upstream data and are sent back to the OLT. Since all light sources are located at the OLT, the requirements for wavelength provisioning and management are relaxed due to a controlled environment. External modulators or reflective SOAs (RSOAs) can be used for upstream modulation. Compared to external modulators, SOAs can provide signal gain for the upstream transmission and, thus, achieve better performance. However, SOAs have limited modulation speed. To transmit downstream and upstream in a full duplex manner in the same wavelength, various data modulation schemes have been proposed, including ASK-ASK using different power offset values, frequencyshift keying-ASK, and SCM-SCM [43]. The disadvantages of CLS are backscattering due to Rayleigh or Brillouin effect and the polarization sensitivity of the RSOAs or optical modulators. Also, the cost of RSOAs or external modulators can be an obstacle for deployment.

Network architectures: CLS architectures were first proposed in [44] and further developed in [45]-[47]. Fig. 10 illustrates a generic CLS architecture. All the light sources are at the OLT. These provide the optical power for downstream and upstream transmission. The downstream transmission case is straightforward. The upstream data transmission works as follows: The transmitters at the OLT provide a carrier at the particular wavelength of an ONU. The ONU modulates this carrier with its queued data and sends it back upstream. A circulator at the OLT directs these upstream data onto an AWG followed by an array of receivers: one for each ONU. Since the traffic in opposite directions is transmitted on the same wavelength and the same fiber, Rayleigh backscattering and reflections need to be minimized throughout the network. To achieve the goals, the optical power of the carrier needs to be high enough for upstream transmission but limited to prevent significant Rayleigh backscattering. Clean connectors throughout the distribution network are required to minimize the reflections.

Fig. 10 illustrates two possible ONU designs. ONU1 has a circulator and a splitter. Incoming data are received by a receiver that does not need to be tunable while the incoming carrier is modulated and sent back. An SOA is used as an amplifier and modulator due to its potential for integration with the electronics of the ONU [45]. ONU2 illustrates a simpler design using a one-port RSOA. Again, the downstream data are received by a photodiode receiver, whereas the upstream data are modulated onto a CW by the RSOA. In [48], the RSOA acts as a detector as well by measuring the voltage difference produced by the change in carrier density inside the cavity.

5) Evolutionary Architectures From TDM-PON to WDM$P O N$ : A transition from TDM-PON to WDM-PON has to take place, if at all possible, without interrupting existing networks and services. To address that requirement, novel architectures enabling smooth evolution from the current generation to the $\mathrm{C}+2$ generation have been proposed. These architectures 


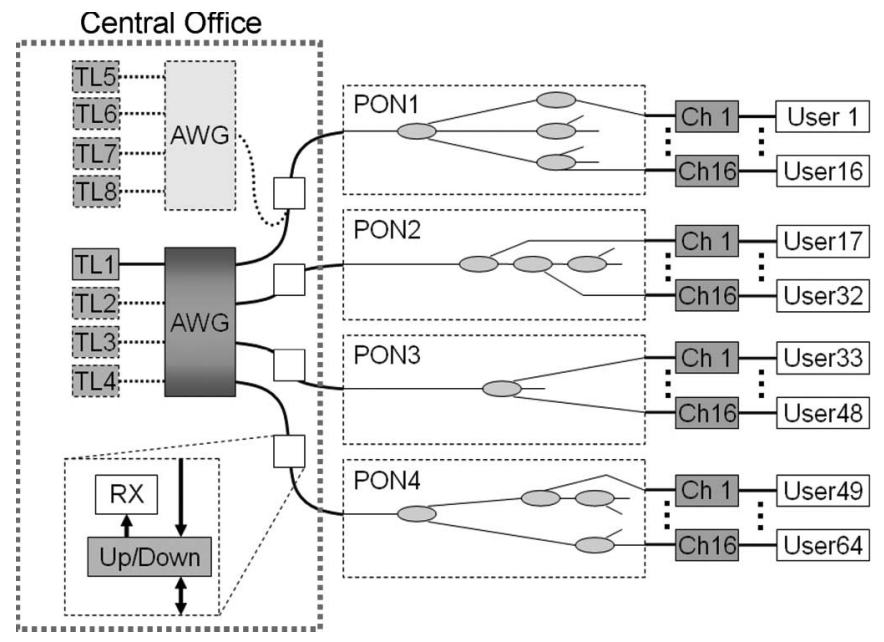

Fig. 11. SUCCESS-DWA architecture.

assume that the deployment of current-generation TDM-PONs will be extensive, and, subsequently, service providers will be reluctant to assume the cost of entirely new WDM-PONs. In this section, we discuss two evolutionary architectures facilitating a graceful migration from TDM-PONs to WDM-PONs.

Stanford University access (SUCCESS) dynamic wavelength allocation (DWA): SUCCESS-DWA PON is an optical access network that employs DWA [49]. SUCCESSDWA PON allows flexible transition from TDM-PON to hybrid TDM/WDM-PON to full WDM-PON, depending on the bandwidth demands. Fig. 11 shows the basic architecture of SUCCESS-DWA PON. The key components of the system are the fast TLs, cyclic AWGs, and thin-film WDM filters. The field-deployed infrastructure is compatible with TDM-PONs; therefore, the existing infrastructure of TDM-PONs remains intact when they are brought together into a SUCCESS-DWA PON. In the basic architecture, a cyclic AWG multiplexes the TLs and routes the TL outputs to physical PONs, depending on the wavelength. Each ONU within a single PON contains a unique fixed-wavelength filter and a burst-mode receiver. The key is that the passband of the ONU filter entirely encompasses the free spectral range of the AWG. For example, 200-GHz ONU filters would work with a $4 \times 4$ cyclic $50-\mathrm{GHz}$ AWG. In this architecture, any TL can individually address any ONU across separate physical PONs at any given time; this results in better statistical multiplexing gain than that of TDM-PONs [50].

Details of design and implementation issues of the key building blocks of SUCCESS-DWA PON, including fast TLs, burstmode receivers, and scheduling algorithms with QoS support, are described in [50]. A testbed is constructed, and videostreaming experiments are performed to show the feasibility of the integrated system. The experimental testbed consists of two fast TLs that are modulated at $1.25 \mathrm{~Gb} / \mathrm{s}$ and multiplexed by an AWG. Four user wavelength channels are defined, and 16 subscribers share bandwidth in wavelength and time domains. Unlike the downstream transmission in a TDM-PON that is continuous, SUCCESS DWA-PON requires burst-mode transmission in the downstream direction. Therefore, burstmode level and clock recovery are implemented in the ONU receiver, and burst transmission is implemented in the OLT by tuning the wavelength of TL. The data framing, coding, and scheduling are performed by the field programmable gate arrays (FPGAs) in the OLT and the ONU [50].

One disadvantage of SUCCESS-DWA is the limit on the total number of users imposed by optical components such as the TLs. In DWA-PON, the required TR of the TL is

$$
\mathrm{TR}=N_{\mathrm{AWG}} \times n \times W
$$

where $n$ is the user number per PON, $N_{\mathrm{AWG}}$ is the port number of the cyclic AWG, and $W$ is the channel width. For example, if $N_{\mathrm{AWG}}=4, W=0.8 \mathrm{~nm}$, and $n=16$, then the required TR will be $51.2 \mathrm{~nm}$. Note that the total user number across the four physical PONs is 64 . As such, the maximum number of users is limited by the TR of the TL and the channel width.

SUCCESS hybrid PON (HPON): Fig. 12 illustrates the transition path from TDM to hybrid WDM/TDM in SUCCESSHPON [47]. In Fig. 12(a), a single CO provides service for three existing TDM-PONs. Each of these requires exclusive cabling and an OLT inside the CO. As a first step, in Fig 12(b), the passive splitters of the TDM-PONs are replaced by RNs consisting of passive couplers and thin-film add/drop filters. The feeder fiber of each TDM-PON is replaced with a single fiber ring that interconnects all the RNs served by this CO. The benefits of this topology evolution in terms of resource sharing and protection are considerable since, now, all the TDM ONUs can be served by the same equipment in the CO. It will also allow serving WDM ONUs using the same network. Importantly, the distribution fibers are not changed in this migration. From the TDM ONUs' point of view, the functionality of the optical access network is exactly the same; therefore, they can continue to exactly operate as before.

As more users demand higher bandwidths, RNs that contain an AWG as a DWDM MUX/DMUX are inserted in the ring, as shown in Fig. 12(c). Each WDM ONU has a dedicated DWDM channel between it and the OLT. To avoid the need for tunable sources at the ONU, CLSs with RSOAs as amplifiers/modulators are used, using intensity modulation in both directions. In this manner, up to $1.25-\mathrm{Gb} / \mathrm{s}$ half-duplex transmission can be provided with current technologies [43]. The main benefits of this network architecture are as follows.

1) It allows for TDM-PONs and WDM-PONs to coexist, making a smooth transition possible.

2) It is cost efficient by sharing the expensive WDM components and using a CLS approach.

3) It gracefully scales by adding more tunable components at the OLT.

To upgrade the capacity even further, the RSOA modulator can be replaced with a stabilized laser source ONU for full-duplex communications.

Novel MAC protocols and scheduling algorithms are needed to enable the efficient sharing of tunable WDM resources among many users for downstream and upstream transmissions. These algorithms include sequential mode, in which the scheduling is done as packets are received or transmitted, and batch mode (batch earliest departure first), in which the scheduling is performed every certain period of time, 
(a)

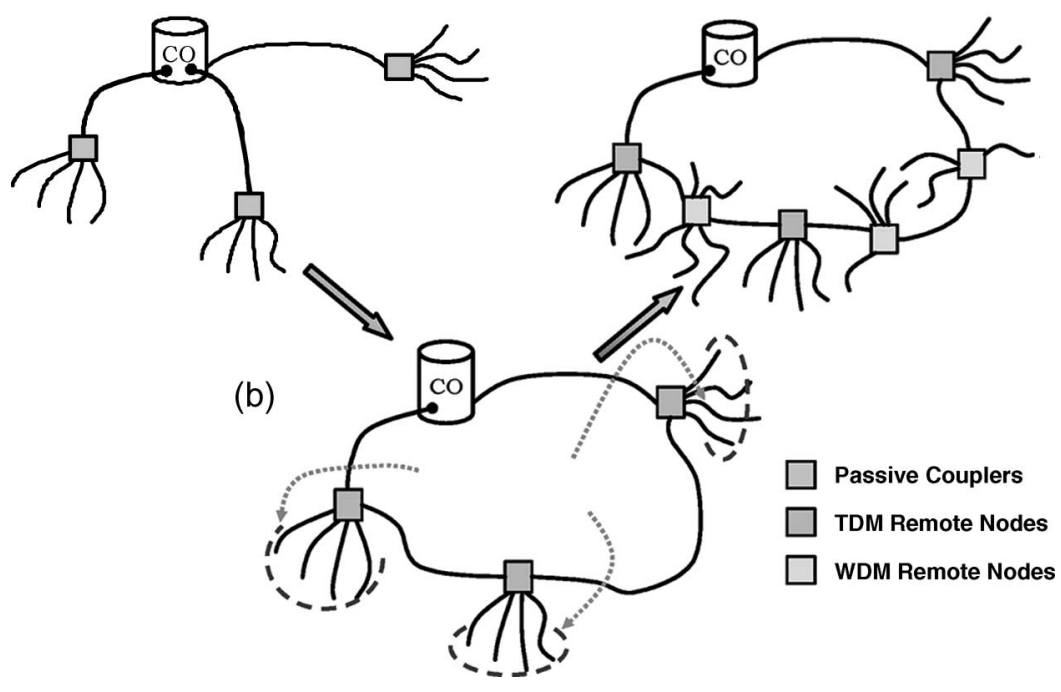

Fig. 12. SUCCESS-HPON transition from TDM to WDM. (a) Legacy TDM-PONs. (b) Multiple legacy TDM-PONs share resources at CO using new RNs. (c) New WDM-PONs for higher bandwidth customers.

TABLE IV

WDM-PON APPROACH COMPARISON SUMMARY

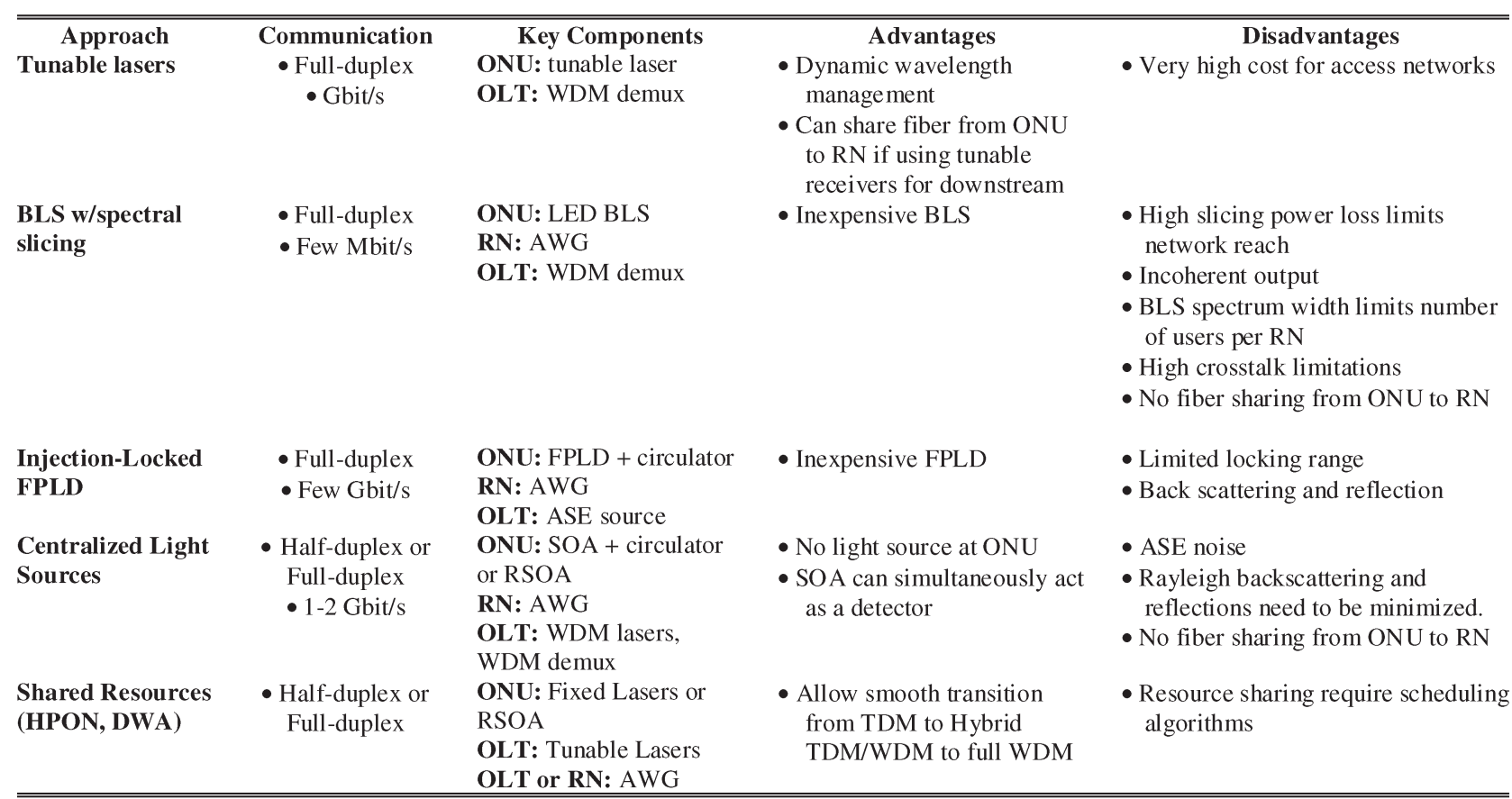

allowing for throughput optimization at the expense of additive delay. The sequential scheduling with schedule-time framing algorithm has relatively low computational complexity, high throughput, and low delay. A comparison of the throughput of these three algorithms is available in [51].

The main disadvantage of SUCCESS-HPON is the need to convert the topology from trees to a ring to enable the support for legacy TDM-PONs in the same network for a smooth transition. However, the scheduling algorithm technology for the CLS approach with shared resources can be used in tree topologies as well.

The $\mathrm{C}+2$ generation technologies and architectures are summarized and compared in Table IV.

\section{BURST-MODE TRANSMISSION IN OPTICAL ACCESS NETWORKS}

In TDM-PON, the downstream traffic is continuously broadcast to all ONUs. Therefore, conventional continuous-mode transmitters can be used in the OLT, and continuous-mode receivers can be used in ONUs. For the upstream link, however, ONU transmitters are active only for their preassigned time slots and off else wise; hence, burst-mode transmitters are required for ONUs. On the OLT side, to avoid traffic collision due to the distance variation of ONUs, ranging is continuously performed, and guard time is inserted between consecutive upstream bursts. To handle the discontinuous and asynchronous bursts with varying optical power levels, burst-mode receivers 
are required at the OLTs. Aside from TDM-PON, burst-mode transceivers are necessary for many hybrid TDM/WDM-PON architectures [47], [49], where the required functionalities are more complicated than those in TDM-PONs. In this section, we review the design challenges of burst-mode transceivers for optical access networks.

\section{A. Burst-Mode Laser Drivers}

A burst-mode transmitter requires the following functionalities: fast burst on/off speed, sufficient power suppression during idle period, and stable, accurate power emission during burst transmission. These functionalities can be performed by specially designed laser drivers that are the most critical part of burst-mode transmitters [52].

Direct modulation is desirable for access networks. In conventional laser drivers, the bias current is maintained constant for continuous transmission. Burst-mode operation, however, requires the bias current to be quickly turned on/off before/after burst is transmitted. The on/off speed directly determines the required guard time and, thus, the transmission efficiency. A design with burst on/off time of $6-13$ bits at $1.25 \mathrm{~Gb} / \mathrm{s}$ has been reported in [53].

The optical power suppression of a burst-mode transmitter during idle periods is critical for TDM-PONs due to their tree topology. Small light emissions from idle ONUs might add up to a significant interference to the signal transmitted by the active ONU [53]. In EPON, the average power emitted by an idle ONU should not exceed $-45 \mathrm{dBm}$.

Stable and accurate output power is essential for PONs since power leveling is employed to equalize the received power from ONUs with different distance from the $\mathrm{CO}$. To monitor the emission power, a photodiode is usually packaged within the transmitter laser, and it feeds the monitored emission back to the laser driver. The power, thus, can be automatically adjusted to compensate for changes in temperature and other parameters using a feedback loop. Unlike conventional laser drivers that use relatively slow control mechanism, the automatic power control for burst-mode laser drivers has to be able to handle rapid transients [53].

\section{B. Burst-Mode Receivers}

A burst-mode receiver needs to have high sensitivity, wide dynamic range, and fast time response to the received bursts. Conventional optical receivers are not suitable for burst-mode detection because they are not able to instantaneously handle bursts with large power difference and random phase. Design challenges for burst-mode receivers include dynamic sensitivity recovery, fast level recovery, and fast clock recovery.

When a weak burst follows a strong burst, it is hard to detect the weak signal. Dynamic sensitivity recovery is necessary for detection of the weak signal. The recovery of the weak burst is limited by photodiode carrier transport effect, amplifier slew and charging rates, and unintentional automatic gain control effects.

For level recovery, feedback and feedforward structures have been reported. In the feedback design, the loop consists of a differential input/output transimpedance amplifier and a peak detection circuit [54]. In the feedforward design, the signal from preamplifier is fed forward into a peak detection circuitry to determine the threshold [55]. In principle, a feedback structure enables the receiver to work more reliably, but a differential input/output preamplifier is needed. A feedforward structure has faster response and can leverage conventional dc-coupled preamplifiers. However, the circuitry needs to be carefully designed to prevent oscillations. Level recovery circuits with simple and robust design and good performance are still an open issue that needs further investigation.

For clock recovery, phase-locked loop (PLL) is widely used in conventional systems. In burst-mode operation, it is not suitable because it usually takes thousands of bits to recover the clock. To enhance the clock recovery speed, feedforward architectures such as gated voltage-controlled oscillator [56] and oversampling [57] have been proposed. However, the jitter tolerance of these architectures is lower than that of the feedback architecture.

The clock recovery design is more involved for hybrid TDM/WDM-PONs. In TDM-PON, if PLL type clock recovery is used, the ONUs keep locking to the continuous downstream signal. As a result, all ONUs lock to the OLT's transmitter clock, and the recovered clock can be leveraged for the upstream transmission. In this way, all the ONUs are synchronized with the OLT, and the only work left for the OLT clock recovery is to rapidly align the local clock phase to the optimum sampling point. In contrast, for hybrid TDM/WDM-PON, the downstream and upstream transmissions are burst mode. As a result, the OLT and the ONUs are independently running at their local clock frequencies. Therefore, the clock recovery design must take into account the inherent frequency difference of each received burst.

\section{TOPICS FOR FUTURE RESEARCH}

In this section, we discuss PON issues that have not been addressed as yet but have important practical implications that merit additional research. Two open research topics for PON systems are security and convergence with wireless technologies.

\section{A. PON Security}

Due to their point-to-multipoint topology, PONs are inherently vulnerable to malicious attacks. The main security threats in current TDM-PONs are 1) denial of service attack, 2) eavesdropping, and 3) masquerading of an ONU. Even for WDM-PON, security could be compromised when splitters are deployed.

A denial of service may occur when an ONU is set to continuously transmit at the upstream wavelength with a highenough power to block one, several, or all ONUs from getting their data through. Such corrupted sources could be either a malfunctioning/promiscuous ONU or a purposely misused upstream laser diode at an ONU. Due to PON's passive nature, it is technically difficult to monitor the attack and address the problem even when it is discovered. Recent proposals [58], [59] have made separate efforts to mitigate such challenges by 
effectively identifying the attackers and disconnecting malicious sources from the network after discovery.

In the downstream direction, a PON behaves as a physical broadcast network. Every frame is delivered to all ONUs in the PON system. Eavesdropping may happen if an ONU is able to listen to the data that are sent to or from another ONU. Though no authentication and encryption mechanism is specified in the standard, many EPON vendors have implemented their own proprietary solutions for downstream transmission. The ITU G.984 GPON standard requires the advanced encryption standard for downstream transmission. Recent experiments have also illustrated that a modified mal-intended ONU can accurately eavesdrop on the upstream traffic due to power reflections of a victimized ONU that shares a passive coupler at the ODN [60]. The study suggests that upstream encryption is also needed to minimize the probability of eavesdropping.

In today's commercial systems, newly connected or offline ONUs can automatically gain access to PON in the registration process [61]. A malicious ONU could pose as the victimized ONU and send multipoint control protocol frames and operation, administration, and maintenance frames in an attempt to steal the bandwidth or attack the system. The operator needs to implement an authentication mechanism as well as to have an ability to effectively identify the users in the access network to avoid masquerading problems.

In the future, quantum encryption might be able to provide the most secure PON communications [62]. However, quantum encryption is still an immature technology that needs further research. The cost and the complexity of implementing quantum encryption in PONs have to be justified by the needs for highly secure services, and that point has not been reached as yet.

\section{B. Hybrid Optical/Wireless Access Networks}

Although optical access networks provide high-bandwidth and reliable service, they require mass deployment of fiberoptics infrastructure to reach numerous end users, which results in significant investment for the operators. In addition, the provisioned connectivity is limited to an area that is covered by local area networks, which are usually homes or small business units. Wireless access networks, on the other hand, require less infrastructure deployment and can provide flexible and ubiquitous access connections for the end users. Therefore, one might envision a hybrid optical/wireless network architecture, in which the optical network provides high-capacity backhaul, and the wireless network provides flexible link to the end users. Such hybrid architecture might emerge as a viable access solution.

Hybrid optical/wireless access networks are proposed in [63] and [64]. In both cases, optical networks are employed as backhaul for Wi-Fi, WiMax, and cellular systems. Reference [63] is focused on hybrid optical/wireless access networks for highly populated areas. It consists of an optical grid network providing broadband backhauling and many wireless mesh networks penetrating into the neighborhoods to provide lowcost ubiquitous links. This hybrid architecture is scalable and flexible to meet the increasing bandwidth demand by increasing the grid and mesh density.

\section{CONCLUSION}

High bandwidth and low cost have made TDM-PON a promising solution to the bandwidth bottleneck in access networks. This solution is being deployed by many service providers around the globe; we classify it as the current (C) generation. Once deployed, C-generation PONs will have to evolve further to satisfy the ever-increasing bandwidth demands from the end users. In this paper, we describe current TDM-PONs and their bandwidth enhancements proposed by standards bodies (IEEE and ITU-T). Such PON systems are designed to provide graceful evolution from standard TDM-PONs; we define such systems as the $\mathrm{C}+1$ generation. Key technologies for $\mathrm{C}+1$ PONs are service overlay based on WDM, SCM, and line coding. Next, we review $\mathrm{C}+2$ generation PON systems, which focus on providing much higher bandwidth to end users in the long term. $\mathrm{C}+2$ PONs are optimized for enhancing system capacity and improving network scalability and protection support. The key enabling technologies for $\mathrm{C}+2$ PONs are burst-mode optical transceivers, WDM light sources, and CLSs. Important topics for future research include PON security and hybrid optical/wireless access networks.

\section{ACKNOWLEDGMENT}

The authors would like to thank Prof. M. Maier for fruitful discussions and insightful comments.

\section{REFERENCES}

[1] K. Fukuchi, T. Kasamatsu, M. Morie, R. Ohhira, T. Ito, K. Sekiya, D. Ogasahara, and T. Ono, "10.92-Tb/s $(273 \times 40-\mathrm{Gb} / \mathrm{s})$ triple-band/ ultra-dense WDM optical-repeatered transmission experiment," in Proc. OFC, 2001, pp. PD24-1-PD24-3.

[2] P. Chanclou, S. Gosselin, J. F. Palacios, V. L. Alvarez, and E. Zouganeli, "Overview of the optical broadband access evolution: A joint article by operators in the IST network of excellence e-Photon/One," IEEE Commun. Mag., vol. 44, no. 8, pp. 29-35, Aug. 2006.

[3] P. E. Green, Jr., "Fiber to the home: The next big broadband thing," IEEE Commun. Mag., vol. 42, no. 9, pp. 100-106, Sep. 2004.

[4] "FTTP: Revolutionizing the Bells' Telecom Networks," Bernstein Res.-Telcordia Technol. Rep., May 2004.

[5] J.-M. Kang and S.-K. Han, "A novel hybrid WDM/SCM-PON sharing wavelength for up- and down-link using reflective semiconductor optical amplifier," IEEE Photon. Technol. Lett., vol. 18, no. 3, pp. 502-504, Feb. 2006.

[6] J.-S. Baik and C.-H. Lee, "Hybrid WDM/SCMA-PON using wavelengthlocked Fabry-Pérot laser diodes," IEEE Photon. Technol. Lett., vol. 18, no. 15, pp. 1585-1587, Aug. 2006.

[7] R. Hirth, "1 Gbps to 10 Gbps migration," in Proc. IEEE 802 Plenary Meeting, San Diego, CA, Jul. 2006.

[8] R. Bavey, J. Kani, F. Bourgart, and K. McCammon, "Options for future optical access networks," IEEE Commun. Mag., vol. 44, no. 10, pp. 50-56, Oct. 2006.

[9] I. Van de Voorde, C. M. Martin, J. Vandewedge, and X. Z. Qiu, "The superPON demonstrator: An exploration of possible evolution paths for optical access networks," IEEE Commun. Mag., vol. 38, no. 2, pp. 74-82, Feb. 2000.

[10] D. Nesset, R. P. Davey, D. Shea, P. Kirkpatrick, S. Q. Shang, M. Lobel, and B. Christensen, " $10 \mathrm{~Gb} / \mathrm{s}$ bidirectional transmission in 1024-way split, $110 \mathrm{~km}$ reach, PON system using commercial transceiver modules, super FEC and EDC," in Proc. ECOC, Sep. 2005, pp. 135-138.

[11] R. P. Davey, P. Healey, I. Hope, P. Watkinson, and D. B. Payne, "DWDM reach extension of a GPON to $135 \mathrm{~km}$," J. Lightw. Technol., vol. 24, no. 1, pp. 29-32, Jan. 2006.

[12] G. C. Gupta, M. Kashima, H. Iwamura, H. Tamai, T. Ushikubo, and T. Kamijoh, "Over 100 km bidirectional, multi-channels COF-PON without optical amplifier," in Proc. OFC, Mar. 2006, pp. 1-3. 
[13] C. Bouchat, C. Dessauvages, F. Fredricx, C. Hardalov, R. Schoop, and P. Vetter, "WDM-upgrade PONs for FTTH and FTTBusiness," in Proc. Int Workshop Opt. Hybrid Access Netw., Florence, Italy, Jun. 2002, pp. 231-238.

[14] Y.-L. Hsueh, M. S. Rogge, W.-T. Shaw, J. Kim, S. Yamamoto, and L. G. Kazovsky, "Smooth upgrade of existing passive optical networks with spectral-shaping line coding service overlay," J. Lightw. Technol., vol. 23, no. 9, pp. 2629-2637, Sep. 2005.

[15] C. Bouchat, C. Dessauvages, F. Fredricx, and P. Vetter, "Feasibility of DWDM-upgraded PON for FTTBusiness and demonstrator," in Proc. Symp. IEEE/LEOS Benelux Chapter, Amsterdam, The Netherlands, 2002, pp. 91-94.

[16] R. D. Feldman, T. H. Wood, J. P. Meester, and R. F. Austin, "Broadband update of an operating narrowband single-fiber passive optical network using coarse wavelength division multiplexing and sub-carrier multiple access," J. Lightw. Technol., vol. 16, no. 1, pp. 1-8, Jan. 1998.

[17] S. L. Woodward et al., "Reduction of optical-beat interference in subcarrier networks," IEEE Photon. Technol. Lett., vol. 8, no. 5, pp. 694-696, May 1995.

[18] T. H. Wood and N. K. Shankaranarayanan, "Operation of a passive optical network with subcarrier multiplexing in the presence of optical beat interference," J. Lightw. Technol., vol. 11, no. 10, pp. 1632-1640, Oct. 1993

[19] M. M. Banat and M. Kavehrad, "Reduction of optical beat interference in SCM/WDMA networks using pseudorandom phase modulation," J. Lightw. Technol., vol. 12, no. 10, pp. 1863-1868, Oct. 1994.

[20] R. D. Feldman et al., "Reduction of optical beat interference in a subcarrier multiple-access passive optical network through the use of an amplified light-emitting diode," IEEE Photon. Technol. Lett., vol. 8, no. 1, pp. 116-118, Jan. 1996

[21] C. C. Hsiao et al., "Multiple access in the presence of optical-beat and co-channel interference using Walsh-code-based synchronized CDMA technique," IEEE Photon. Technol. Lett., vol. 9, no. 8, pp. 1173-1175, Aug. 1997.

[22] C. Lee, H. Lee, D. Kim, and H. Jung, "A new line code for 10-Gigabit Ethernet: MB810," in Proc. IEEE Int. Conf. Commun., New Orleans, LA, Jun. 2000, vol. 3, pp. 1774-1777.

[23] G. L. Pierobon, "Codes for zero spectral density at zero frequency," IEEE Trans. Commun., vol. COM-30, no. 2, pp. 435-439, Mar. 1984.

[24] C. G. Lee, J. Cho, C. H. Zhung, J. H. Ko, and D. Y. Kim, "A test result of minimum-bandwidth line code MB34 in 10 Gbps optical transmission system," in Proc. Int. Conf. Inf., Commun., Signal Process., Singapore, Sep. 1997, pp. 1148-1152.

[25] A. X. Widmer and P. A. Franaszek, "A DC-balanced, portioned lock, 8B/10B transmission code," IBM J. Res. Develop., vol. 27, no. 5, pp. 440451, Sep. 1983.

[26] G. Maier, M. Martinelli, A. Pattavina, and E. Salvadori, "Design and cost performance of the multistage WDM-PON access networks," J. Lightw. Technol., vol. 18, no. 2, pp. 125-143, Feb. 2000.

[27] J. Prat, P. E. Balaguer, J. M. Gené, O. Díaz, and S. Figuerola, Fiber-tothe-Home Technologies. Boston, MA: Kluwer, 2002.

[28] P.-J. Rigole, S. Nilsson, L. Backbom, T. Klinga, J. Wallin, B. Stalnacke, E. Berglind, and B. Stoltz, "Access to 20 evenly distributed wavelengths over $100 \mathrm{~nm}$ using only a single current tuning in a four-electrode monolithic semiconductor laser," IEEE Photon. Technol. Lett., vol. 7, no. 11, pp. 1249-1251, Nov. 1995.

[29] C. J. Chang-Hasnain, "Tunable VCSEL," IEEE J. Sel. Topics Quantum Electron., vol. 6, no. 6, pp. 978-987, Nov./Dec. 2000

[30] R. Chen, H. Chin, D. A. B. Miller, K. Ma, and J. S. Harris, Jr., "MSMbased integrated CMOS wavelength-tunable optical receiver," IEEE Photon. Technol. Lett., vol. 17, no. 6, pp. 1271-1273, Jun. 2005.

[31] S. S. Wagner and T. E. Chapuran, "Broadband high-density WDM transmission using superluminescent diodes," Electron. Lett., vol. 26, no. 11, pp. 696-697, May 1990.

[32] D. K. Jung, H. Kim, K. H. Han, and Y. C. Chung, "Spectrum-sliced bidirectional passive optical network for simultaneous transmission of WDM and digital broadcast video signals," Electron. Lett., vol. 37, no. 5, pp. 308-309, Mar. 2001

[33] S. L. Woodward, P. P. Iannone, K. C. Reichmann, and N. J. Frigo, "A spectrally sliced PON employing Fabry-Pérot lasers," IEEE Photon. Technol. Lett., vol. 10, no. 9, pp. 818-820, Sep. 1997.

[34] M. H. Reeve, A. R. Hunwicks, W. Zhao, S. G. Methley, L. Bickers, and S. Hornung, "LED spectral slicing for single-mode local loop applications," Electron. Lett., vol. 24, no. 7, pp. 389-390, Mar. 1988.

[35] M. Zirngibl, C. H. Joyner, L. W. Stulz, C. Dragone, H. M. Presby, and I. P. Kaminow, "LARnet, a local access router network," IEEE Photon. Technol. Lett., vol. 7, no. 2, pp. 215-217, Feb. 1995.
[36] D. K. Jung, S. K. Shin, C.-H. Lee, and Y. C. Chung, "Wavelengthdivision-multiplexed passive optical network based on spectrum-slicing techniques," IEEE Photon. Technol. Lett., vol. 10, no. 9, pp. 1334-1336, Sep. 1998.

[37] R. D. Feldman, "Crosstalk and loss in wavelength division multiplexed systems employing spectral slicing," J. Lightw. Technol., vol. 15, no. 10, pp. 1823-1831, Oct. 1997.

[38] S. Kobayashi, J. Yamada, S. Machida, and T. Kimura, "Single-mode operation of $500 \mathrm{Mb} / \mathrm{s}$ modulated AlGaAs semiconductor laser by injection locking," Electron. Lett., vol. 16, no. 19, pp. 746-748, Sep. 1980.

[39] S.-M. Lee, K.-M. Choi, S.-G. Mun, J.-H. Moon, and C.-H. Lee, "Dense WDM-PON based on wavelength-locked Fabry-Pérot laser diodes," IEEE Photon. Technol. Lett., vol. 17, no. 7, pp. 1579-1581, Jul. 2005.

[40] L. Y. Chan, C. K. Chan, D. T. K. Tong, F. Tong, and L. K. Chen, "Upstream traffic transmitter using injection-locked Fabry-Pérot laser diode as modulator for WDM access networks," Electron. Lett., vol. 38, no. 1 , pp. 43-44, Jan. 2002.

[41] F.-T. An, K. S. Kim, Y.-L. Hsueh, M. Rogge, W.-T. Shaw, and L. G. Kazovsky, "Evolution, challenges and enabling technologies for future WDM-based optical access networks," in Proc. 2nd Symp. Photon., Netw. Comput., Cary, NC, Sep. 2003.

[42] D. J. Shin, Y. C. Keh, J. W. Kwon, E. H. Lee, J. K. Lee, M. K. Park, J. W. Park, Y. K. Oh, S. W. Kim, I. K. Yun, H. C. Shin, D. Heo, J. S. Lee, H. S. Shin, H. S. Kim, S. B. Park, D. K. Jung, S. Hwang, Y. J. Oh, D. H. Jang, and C. S. Shim, "Low-cost WDM-PON with colorless bidirectional transceivers," J. Lightw. Technol., vol. 24, no. 1, pp. 158-165, Jan. 2006.

[43] C. Arellano, C. Bock, and J. Prat, "RSOA-based optical network units for WDM-PON," presented at the Optical Fiber Commun. Conf. (OFC), Anaheim, CA, 2006, Paper OtuC1.

[44] N. J. Frigo, P. P. Iannone, P. D. Magill, T. E. Darcie, M. M. Downs, B. N. Desai, U. Koren, T. L. Koch, C. Dragone, H. M. Presby, and G. E. Bodeep, "A wavelength-division multiplexed passive optical network with cost-shared components," IEEE Photon. Technol. Lett., vol. 6, no. 1, pp. 1365-1367, Nov. 1994.

[45] J. Kani, M. Teshima, K. Akimoto, N. Takachio, H. Suzuki, K. Iwatsuki, and M. Ishii, "A WDM-based optical access network for wide-area gigabit access services," IEEE Commun. Mag., vol. 41, no. 2, pp. S43-S48, Feb. 2003.

[46] H. Takesue and T. Sugie, "Wavelength channel data rewrite using saturated SOA modulator for WDM networks with centralized light sources,' J. Lightw. Technol., vol. 21, no. 11, pp. 2546-2556, Nov. 2003.

[47] F.-T. An, K. S. Kim, D. Gutierrez, S. Yam, E. Hu, K. Shrikhande, and L. G. Kazovsky, "SUCCESS: A next-generation hybrid WDM/TDM optical access network architecture," J. Lightw. Technol., vol. 22, no. 11, pp. 2557-2569, Nov. 2004

[48] J. Prat, C. Arellano, V. Polo, and C. Bock, "Optical network unit based on a bidirectional reflective semiconductor optical amplifier for fiber-to-thehome networks," IEEE Photon. Technol. Lett., vol. 17, no. 1, pp. 250-252, Jan. 2005.

[49] Y.-L. Hsueh, M. S. Rogge, S. Yamamoto, and L. G. Kazovsky, "A highly flexible and efficient passive optical network employing dynamic wavelength allocation," J. Lightw. Technol., vol. 23, no. 1, pp. 277-286, Jan. 2005.

[50] Y.-L. Hsueh, W.-T. Shaw, L. G. Kazovsky, A. Agata, and S. Yamamoto, "SUCCESS PON demonstrator: Experimental exploration of next-generation optical access networks," IEEE Commun. Mag., vol. 43, no. 8, pp. S26-S33, Aug. 2005.

[51] K. S. Kim, D. Gutierrez, F.-T. An, and L. G. Kazovsky, "Design and performance analysis of scheduling algorithms for WDM-PON under SUCCESS-HPON architecture," J. Lightw. Technol., vol. 23, no. 11, pp. 3716-3731, Nov. 2005.

[52] J. Bauwelinck, W. Chen, D. Verhulst, Y. Martens, P. Ossieur, X.-Z. Qiu, and J. Vandewege, "A high-resolution burst-mode laser transmitter with fast and accurate level monitoring for $1.25 \mathrm{~Gb} / \mathrm{s}$ upstream GPONs," IEEE J. Solid-State Circuits, vol. 40, no. 6, pp. 1322-1330, Jun. 2005.

[53] X.-Z. Qiu, P. Ossieur, J. Bauwelinck, Y. Yi, D. Verhulst, J. Vandewege, B. De Vos, and P. Solina, "Development of GPON upstream physical-media-dependent prototypes," J. Lightw. Technol., vol. 22, no. 11 , pp. 2498-2508, Nov. 2004

[54] S. Brigati, P. Colombara, L. D'Ascoli, U. Gatti, T. Kerekes, and P. Malcovati, "A SiGe BiCMOS burst-mode $155 \mathrm{Mb} / \mathrm{s}$ receiver for PON," IEEE J. Solid-State Circuits, vol. 37, no. 7, pp. 887-893, Jul. 2002.

[55] M. Nakamura, Y. Imai, Y. Umeda, J. Endo, and Y. Akatsu, "1.25-Gb/s burst-mode receiver ICs with quick response for PON systems," IEEE J. Solid-State Circuits, vol. 40, no. 12, pp. 2680-2688, Dec. 2005. 
[56] P.-U. Han and W.-Y. Choi, "1.25/2.5-Gb/s burst-mode clock recovery circuit with a novel dual bit-rate structure in 0.18-mm CMOS," in Proc. Circuit Syst., 2006, pp. 3069-3072.

[57] S. Vatannia, P.-H. Yeung, and C. Ju, "A fast response 155-Mb/s burstmode optical receiver for PON," IEEE Photon. Technol. Lett., vol. 17, no. 5, pp. 1067-1069, May 2005.

[58] Y. Horiuchi and N. Edagawa, "ONU authentication technique using loopback modulation within a PON disturbance environment," presented at the Optical Fiber Commun. Conf. (OFC), Anaheim, CA, 2005, Paper OFI3.

[59] S.-W. Wong, W.-T. Shaw, S. Das, and L. G. Kazovsky, "Enabling security countermeasure and service restoration in passive optical networks," in Proc. IEEE Globecom, Dec. 2006, pp. 1-5.

[60] D. Gutierrez, J. Cho, and L. G. Kazovsky, "TDM-PON security issues: Upstream encryption is needed," in Proc. OFC, 2007.

[61] Y. Meng, T. Jiang, and D. Xiao, "Analysis and solutions of security issue in Ethernet PON," Proc. SPIE, vol. 5626, pp. 391-399, 2005.

[62] P. D. Kumavor, A. C. Beal, S. Yelin, E. Donkor, and B. C. Wang, "Comparison of four multi-user quantum key distribution schemes over passive optical networks," J. Lightw. Technol., vol. 23, no. 1, pp. 268-276, Jan. 2005.

[63] W.-T. Shaw, D. Gutierrez, K. S. Kim, N. Cheng, S.-H. Wong, S.-H. Yen, and L. G. Kazovsky, "GROW-Net-A hybrid optical wireless access network," in Proc. 9th Joint Conf. Inf. Sci. (Invited Paper), Kaohsiung, Taiwan, R.O.C., Oct. 2006

[64] S. Sarkar, B. Mukherjee, and S. Dixit, "Optimum placement of multiple optical network units (ONUs) in optical-wireless hybrid access networks," in Proc. OFC, Mar. 2006.

[65] B. Ahn and Y. Park, "A symmetric-structure CDMA-PON system and its implementation," IEEE Photon. Technol. Lett., vol. 14, no. 9, pp. 13811383, Sep. 2002.

[66] G. C. Gupta, M. Kashima, H. Iwamura, H. Tamai, T. Ushikubo, and T. Kamijoh, "Over $100 \mathrm{~km}$ bidirectional, multi-channels COF-PON without optical amplifier," in Proc. OFC, Mar. 2006, pp. 1-3.

[67] H. D. Kim, S.-G. Kang, and C.-H. Lee, "A low-cost WDM source with an ASE injected Fabry-Perot semiconductor laser," IEEE Photon. Technol. Lett., vol. 12, no. 8, pp. 1607-1069, Aug. 2000.

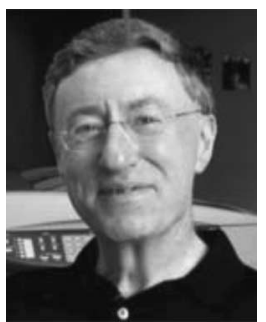

Leonid G. Kazovsky (M'80-SM'83-F'91) received the M.S. and Ph.D. degrees in electrical engineering from the Leningrad Electrotechnical Institute of Communications, Leningrad, Russia, in 1969 and 1972 , respectively.

$\mathrm{He}$ was with Bellcore (now Telcordia), Piscataway, NJ, doing research on WDM and highspeed and coherent optical fiber communication systems. While on Bellcore assignments or sabbaticals with Stanford University, Stanford, CA, he was also with the Heinrich Hertz Institute, Berlin, Germany; Hewlett-Packard Research Laboratories, Bristol, U.K.; Technical University of Eindhoven, Eindhoven, The Netherlands; and Scuola Superiore Sant'Anna, Pisa, Italy. Since 1990, he has been with Stanford University, where he founded Photonics and Networking Research Laboratory (PNRL) and has been leading the PNRL since then. Through research contracts, consulting engagements, and other arrangements, he worked with many industrial companies and U.S. government agencies including Sprint, Digital Equipment Corporation, General Telephone and Electronics, AT\&T, Institutional Venture Partners, Lucent, Hitachi, Kokusai Denshin Denwa, Furukawa, Fujitsu, Optivision, and Perimeter on the industrial side and the National Science Foundation (NSF), the Defense Advanced Research Projects Agency, the U.S. Air Force, Navy, and Army, and the Ballistic Missile Defense Organization on the government side. He also helped to launch several startup companies in the Silicon Valley. He was the author or coauthor of two books, some 190 journal technical papers, and 265 conference papers.

Prof. Kazovsky serves or has served on the Editorial Boards of leading journals (IEEE TRANSACTIONS ON COMMUNICATIONS, IEEE PHOTONICS TeChNology LetTers, Wireless Networks) and on Program Committees of leading conferences [Optical Fiber Communications Conference (OFC), Conference on Lasers and Electro-Optics, IEEE Lasers and Electro-Optics Society, International Society For Optical Engineering, and Global Telecommunications Conference]. He has also served as a reviewer for various IEEE and IEE transactions, proceedings, and journals; funding agencies (NSF, OFC, Environmental Research Center, Nuclear Regulatory Commission, etc.); and publishers (Wiley, MacMillan, etc.). He is a Fellow of the Optical Society of America.

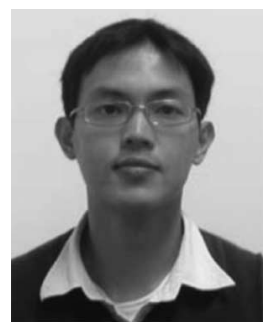

Wei-Tao Shaw received the B.S. degree in electrical engineering from the National Chung-Cheng University, Chia-Yi, Taiwan, R.O.C., in 1999 and the M.S. degree in 2003 from Stanford University, Stanford, CA, where he is currently working toward the Ph.D. degree under the supervision of Prof. L.G. Kazovsky.

His research interests include next-generation optical access networks, hybrid optical-wireless access networks, and circuit design for optical communications systems.

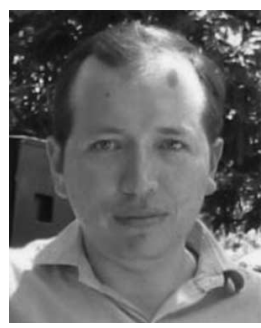

David Gutierrez received the B.Sc. degree in electrical engineering from the Universidad de los Andes, Bogota, Colombia, in 1998 and the M.Sc. degree in electrical engineering in 2002 from Stanford University, Stanford, CA, where he is currently working toward the Ph.D. degree in Prof. L.G. Kazovsky's Photonics and Networking Research Laboratory.

His current research focuses on access and metro network architectures, protocols, and algorithms.

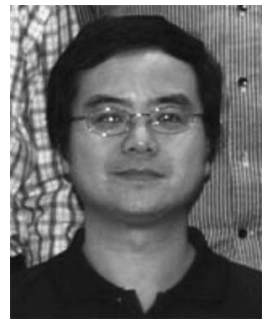

Ning Cheng received the B.Eng. degree in electronic engineering from Xi'an Jiaotong University, Xi'an, China, in 1992, the M.Eng. degree in electronic engineering from Southeast University, Nanjing, China, in 1995, and the Ph.D. degree in electrical engineering from Queen's University, Kingston, ON, Canada, in November 2003.

$\mathrm{He}$ is currently a Postdoctoral Research Fellow with the Department of Electrical Engineering, Stanford University, Stanford, CA. His research interests include optical networks, lightwave systems, and photonic devices. With over ten years of research experience, he has contributed to over 50 papers in photonic technologies.

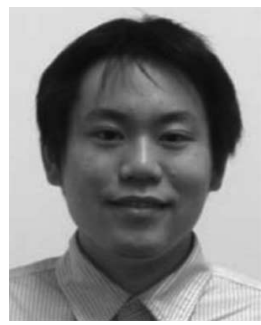

Shing-Wa Wong received the B.S. degree from the University of California, Los Angeles, in 2004 and the M.S. degree in 2006 from Stanford University, Stanford, CA, where he is currently working toward the $\mathrm{Ph} . \mathrm{D}$. degree in electrical engineering.

His interests include design, modeling, and experimental demonstration of next-generation optical access networks and the characterization of highspeed optoelectronic devices and circuits. His other experience includes next-generation PON development, distributed and parallel computing, and wireless MIMO space-time coding. 